\title{
Robust Optimization Framework for Process Parameter and Tolerance Design
}

\author{
Fernando P. Bernardo and Pedro M. Saraiva \\ Dept. of Chemical Engineering, University of Coimbra, 3000 Coimbra, Portugal
}

\begin{abstract}
This article introduces a framework for including different uncertainties at the chemical plant design stage. Through an integrated robust optimization approach and problem formulation, equipment, operating, control, and quality costs are simultaneously taken into account, leading to system, parameter, and tolerance design. Rather than using single pointwise solutions in the decision space, operating windows leading to overall best performance are identified and defined. Such windows and their width allow us to point out control needs and goals at a very early stage of plant design. Two small-scale case studies (for a CSTR and a batch distillation column) provide enough evidence to support the practicality of the optimization framework: the robust solutions found are different and much better than the corresponding solutions obtained with the fully deterministic optimization paradigms.
\end{abstract}

\section{Introduction}

Process control, parameter, and tolerance design issues are not explicitly taken into account at the design stage for most chemical plants. Traditionally, design and control are treated in a sequential way with the following two steps:

(1) Design stage-definition and sizing of equipment and determination of the operating nominal point (usually based on steady-state models).

(2) Control stage-choice and design of the control system based on the operating nominal point determined in the first stage (taking into account dynamic issues).

Furthermore, design stage calculations are traditionally made under deterministic optimization paradigms: an economic objective function is optimized and leads to a pointwise solution in the decision space, without taking into consideration different sorts of uncertainty. Designing a control system based on this solution may be difficult or even impossible since the approach does not consider operability aspects, such as controllability and flexibility.

As Saraiva and Stephanopoulos (1992) and Saraiva (1993, 1996) have shown in previous studies, by considering this traditional approach one ignores the fact that operating decision variables behave as random variables and are always associated with some variability. No matter how good control systems happen to be, in reality ranges of values for the deci-

Correspondence concerning this article should be addressed to P. M. Saraiva. sion variables (concentrations, pressures, flows, etc.) will always occur, eventually bounded within a narrow, but not null, operation window.

As a consequence of not taking into account this processinherent uncertainty, the final solutions found by the conventional approach may be suboptimal: the decision-space zone that surrounds the best deterministic pointwise solution does not correspond in general to the zone of the decision space where best average performance can be achieved.

Besides randomness of operating variables, the deterministic approach also ignores other uncertainties (Pistikopoulos, 1995) such as model-inherent uncertainty (kinetic constants, physical properties, thermodynamic parameters) or external uncertainty (feedstream availability, product demands, economic indexes, environmental regulations).

Several researchers have recently attempted to integrate process design and control. Luyben and Floudas (1994) combine economic and control objectives through a multiobjective optimization framework, incorporating open-loop controllability measures in the mathematical formulation of process synthesis. Bahri et al. $(1996,1997)$ present a methodology for considering flexibility and controllability in process design, where both steady-state and dynamic cases are considered. Mohideen et al. (1996) approach the problem of optimal design for dynamic systems under uncertainty considering both flexibility and control aspects, with the best control structure being proposed through multiloop controllers. 
Process design under uncertainty has also been studied in the literature: flexibility analysis is one of the research directions taken to deal with uncertainty at the design stage (Swaney and Grossmann, 1985; Grossmann and Floudas, 1987; Straub and Grossmann, 1990; Straub, 1991). Pistikopoulos and Ierapetritou (1995) introduced an approach involving stochastic parameters for optimal process design that maximizes an expected revenue while simultaneously measuring design feasibility.

In the context of quality engineering, a common approach for dealing with parameter design is based on running statistically designed experiments and treating the results obtained in order to find robust operating points (Taguchi, 1986; Phadke, 1989; Schmidt and Launsby, 1992; Czitrom and Spagon, 1997; Taylor, 1991). This approach is not adequate for an early stage of plant design, or when reliable process models are available. Diwekar and Rubin (1991, 1994) presented a stochastic optimization framework for implementation of parameter design in chemical processes. In this methodology, uncertain parameters are described by probability density functions (PDFs) and a sampling technique is used to estimate objective function expected values.

This article combines and covers concepts from the broad spectrum of research areas mentioned above (integration of process design and control, process design under uncertainty, and quality engineering). In particular, we will expand on ideas previously developed in the context of process operations (Saraiva 1993, 1996) in order to define and apply a robust stochastic optimization process design framework that combines system, parameter, and tolerance design as they were defined by Taguchi (1986) in his approach to quality engineering.

Our main goal is to determine the optimal design together with the best zone in the operating decision variables space to run the plant, while maximizing an economic objective function that integrates equipment, operating, control and quality costs. Several kinds of uncertainty are taken into account, with expected values of the objective function being estimated through an efficient sampling technique.

The suggested new formulation maximizes overall process performance from an economic point of view, exploring trade-offs between pointwise profitability and robustness (Taguchi, 1986). That is the reason why we will designate our final solution as a robust solution, which represents the optimal balance between process tolerances (reflected in control costs) and output variabilities (reflected in quality costs). Thus, control aspects are also incorporated in the formulation (without considering dynamic behavior) and the robust solution corresponds to an operating region, defined as a set of windows for each operating variable, that identifies control needs and goals, conveying important information for the control system designer.

Therefore, the major contributions of the proposed stochastic framework can be stated as follows:

(1) It leads to the definition of optimal operating regions accounting for control costs and integrates process control issues at an early process design stage.

(2) It considers robustness aspects, the intrinsic random nature of process operating variables, and a wide range of uncertainty sources, enlarging considerably the scope of ran- domness usually incorporated in the field of stochastic process design.

(3) It combines in an integrated way system, parameter, and tolerance design under a common stochastic optimization framework that explores existing plant models, bringing some important quality engineering concepts into the field of process design.

The remaining parts of this article are structured as follows:

- First, we present the design problem statement, clarify its meaning (by way of a CSTR example) and develop its mathematical formulation, leading to a stochastic optimization problem definition.

- Then, a generalized framework is proposed to solve the optimization problem, by coupling a stochastic process model with an external optimization procedure.

- Finally, the relevance of the approach is illustrated through its application to some chemical process design examples, including a CSTR and a batch distillation column.

\section{Problem Statement}

The design problem that we want to address may be defined as follows.

Given a process model described as a set of equations, a set of constraints for feasible plant operation, and appropriate probability density functions for several different uncertainty sources, we want to find the best operating region to run the plant, coupled together with the corresponding design variables' optimal values.

To achieve such a goal, an objective function that is able to integrate equipment, operating, control, and quality costs must be assumed, exploring the trade-offs between these several cost categories.

In order to clarify this problem statement and its objectives, let's consider the CSTR previously studied by Diwekar and Rubin (1994), where a sequential reaction system $(A \rightarrow$ $B \rightarrow C$ ) takes place (Figure 1). The system is described by the stationary relationships of material and energy, with five operating variables (volumetric flow rate, $F$; rate of heat removal, $Q$; inlet concentrations of $A$ and $B, C_{A 0}$ and $C_{B 0}$;

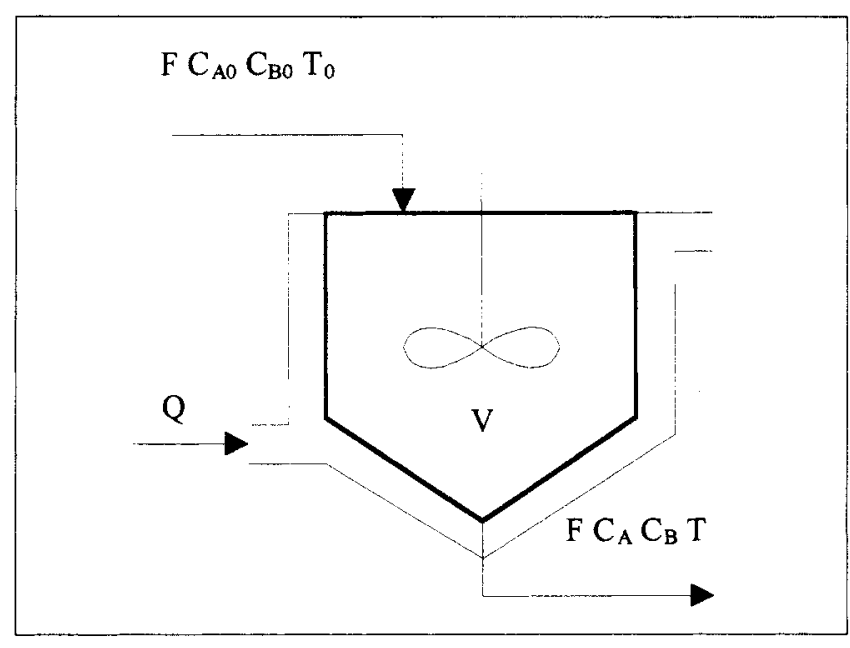

Figure 1. CSTR. 
and inlet temperature, $T_{0}$ ) that are subject to uncertainty. Our design objective is to find the best operating region (in the five variables-space just mentioned) together with the corresponding optimal reactor volume. The determination of such a solution will take simultaneously into account equipment, operating, control, and quality costs, being formulated as a stochastic optimization problem.

In our mathematical formulation, the design objective function is assumed to be an overall annual plant cost $(C)$ that considers equipment cost $\left(C_{d}\right)$, operating cost $\left(C_{o}\right)$, control cost $\left(C_{z}\right)$, and quality cost $\left(C_{s}\right)$.

$$
C=C_{d}+C_{o}+C_{z}+C_{s}
$$

Only equipment costs are considered to have no uncertainties associated with them (since equipment size, dimensions, and materials are considered to be fully deterministic variables). Remaining costs are influenced by the random nature of process variables and parameters. Control costs grow as the tolerances for controlled variables become smaller. Quality costs, expressed by Taguchi (1986) loss functions, increase both with the variability of process outputs that correspond to product quality characteristics and the deviation of their mean from desired nominal target values. Operating costs also depend upon nominal set points of process variables and on their associated tolerances.

Taking into account all uncertainty effects, we will establish overall design decisions through an integrated determination of equipment dimensions and operating zones (defined by both nominal and tolerance values associated with each process variable). Let's suppose that a process model

$$
h\left(d, z, y_{s}, \theta\right)=0
$$

is available, with the following set of constraints

$$
g\left(d, z, y_{s}, \theta\right) \leq 0
$$

where $h$ represents the model equations vector and $g$ the model restrictions vector. The vectors of variables are

$d=$ deterministic equipment design variables (dimensions, materials, etc.);

$z=$ operating decision random variables (concentrations, flows, temperatures, pressures, etc.);

$\theta=$ process model parameters, also subject to uncertainties;

$y_{s}=$ quality-related variables, with associated Taguchi loss functions.

A broad scope of situations can be handled and covered under the above formulation, including the following possibilities:

- The process model does not have to be a steady-state model, but rather may also represent a distributed parameter or even a transient model, since it can be expressed as an input-output model such as $y_{s}=h^{\prime}(d, z, \theta)$, where $h^{\prime}$ defines implicitly the integration of differential equations.

- If a plant configuration is not yet available, the design variables, $d$, will include binary decision variables based on a superstructure that defines all the possible process flowsheet configurations, thus corresponding to a synthesis/design problem.

The uncertainties in $z$ and $\theta$ are described by probability density functions (PDFs) $I(z)$ and $J(\theta)$, which lead to the definition of the corresponding space regions:

$$
\begin{aligned}
& Z=\{z: z \in I(z)\} \\
& T=\{\theta: \theta \in J(\theta)\}
\end{aligned}
$$

If the density functions $I(z)$ or $J(\theta)$ have infinite domains, they must be truncated in order to identify finite $Z$ or $T$ regions. The joint PDF $I J(z, \theta)$, which may lead to the definition of the corresponding space region

$$
Z T=\{(z, \theta): z \in I(z) \wedge \theta \in J(\theta)\}
$$

when propagated through the process model, generates a probability density function $K\left(y_{s}\right)$ and the associated region

$$
Y_{s}=\left\{y_{s}: y_{s} \in K\left(y_{s}\right)\right\}
$$

Putting Eqs. 1-7 together results in the following stochastic optimization problem (P) formulation:

$$
\begin{gathered}
\min _{d, Z} C\left(d, Z, Y_{s}\right)=C_{d}(d)+C_{o}(Z)+C_{z}(Z)+C_{s}\left(Y_{s}\right) \\
\text { s.t. } P\left(h\left(d, z, y_{s}, \theta\right)=0\right)=1, \quad \forall z \in Z, \quad \forall \theta \in T \\
P\left(g\left(d, z, y_{s}, \theta\right) \leq 0\right) \geq \alpha, \quad \forall z \in Z, \quad \forall \theta \in T \\
Z=\{z: z \in I(z)\} \\
T=\{\theta: \theta \in J(\theta)\} \\
Y_{s}=\left\{y_{s}: y_{s} \in K\left(y_{s}\right)\right\}
\end{gathered}
$$

The decision variables are the design variables, $d$, and operating regions, $Z$; model equations are satisfied for all $z$ and $\theta$ values in the $Z$ and $T$ regions, respectively, so that $P\left(h\left(d, z, y_{s}, \theta\right)=0\right)=1 ; \alpha$ is the minimum value required by the designer for the probability of feasible operation under existing uncertainties, that is, the minimum required stochastic flexibility (Straub and Grossmann, 1990).

This problem formulation $(P)$ leads to a final optimal robust solution $\left[d^{*}, Z^{*}\right]$ that defines equipment sizes together with the best zone $Z^{*}$ of the operating decision variables space to run the plant (identified by the combined determination of nominal set points and the associated tolerances for the $z$ variables).

The operating region $Z$ is given as a set of operating windows, $Z_{z}$, for each random variable $z$ :

$$
\begin{aligned}
& Z_{z}=\mu_{z}\left(1 \pm E_{z}\right)=\left[z^{L} ; z^{U}\right] \\
& z^{U}=\mu_{z}\left(1+E_{z}\right) \text { and } z^{L}=\mu_{z}\left(1-E_{z}\right)
\end{aligned}
$$

where $\mu_{z}$ stands for the mean value of $z$ and $E_{z}$ for the associated tolerance.

The definition of $E_{z}$ depends on the probability density function used to describe the random behavior of $z$. When $z$ 
is described by a normal distribution, we will consider:

$$
E_{z}=3.09 \frac{\sigma_{z}}{\mu_{z}}
$$

so that $P(z \in Z)=0.998$.

If other types of probability density functions are used, appropriate similar tolerance definitions can be assumed.

In the above formulation, two things occur. The economic objective function, $C$, properly explores interactions between several cost categories, set points, and tolerances, including process robustness in the sense of Taguchi (1986) and control costs. Second, the optimal robust solution, $Z^{*}$, defines operating set points and tolerances that result from considering in an integrated way different uncertainty sources, as well as control difficulties already known at this early design stage.

The above remarks, it must be noted, stem from an approach that explores only nondynamic plant behavior. This is due to two factors:

(1) Simplicity - the application of a stochastic dynamic formulation to large-scale industrial problems would result in time-consuming and hard-to-solve optimization problems.

(2) Reliability-dynamic considerations at such an early design stage decrease solution reliability due to additional problem complexity. Usually, not enough information is available at this point for studying plant dynamics in an efficient way.

Although we will not study this issue in the forthcoming case studies, flexibility requirements and trade-offs can be evaluated by varying the parameter $\alpha$ and studying the impact over the optimal robust solution found to analyze relationships between flexibility and overall plant cost $(C)$.

The direct solution of problem $(P)$ would derive from the calculation of overall annual cost expected values:

$$
C=\frac{\int_{Z} \int_{T} C_{s}(d, z, \theta) I J(z, \theta) d \theta d z}{\int_{Z} \int_{T} I J(z, \theta) d \theta d z}
$$

In the next section we will present a methodology for solving this problem (P), which uses an efficient sampling technique to compute $C$ estimates.

\section{Optimization Methodology}

The optimization problem (P) defined in the previous section is solved by coupling a stochastic process model with an external optimization procedure (Figure 2). Saraiva (1993, 1996) used a similar approach for identifying robust operating regions from available plant data, while Diwekar and $\mathrm{Ru}$ bin (1994) followed the same methodology for achieving parameter design. In this article, we expand on such previous techniques for solving in an integrated way both parameter and tolerance design problems, since both nominal and tolerance values for the operating variables, $z$, are determined simultaneously. Furthermore, and as stated before, different sources of uncertainty and types of costs are accounted for, leading to a new process design framework that combines and puts together important concepts from process control inte- gration at an early design stage, stochastic process design, and quality engineering.

In the stochastic process model, a sampling technique is used to generate a specified number of observations, $N_{s}$, taken from the joint probability distribution $I J(z, \theta)$. Each $\left[z_{i}, \theta_{i}\right]$ pair generated this way is propagated through the deterministic model $h\left(d, z, y_{s}, \theta\right)$ to compute the corresponding values for $y_{s}, C_{s}\left(y_{s, i}\right), C_{o}\left(z_{i}\right)$ and $g\left(d, z_{i}, y_{s, i}, \theta_{i}\right)$.

After computing these values for all the $N_{s}$ cases, expected values for $C_{s}$ and $C_{o}$ are estimated by the sample mean val. ues:

$$
E\left(C_{s}\right)=\frac{\int_{Z} \int_{T} C_{s}(d, z, \theta) I J(z, \theta) d \theta d z}{\int_{Z} \int_{T} I J(z, \theta) d \theta d z} \equiv \frac{\sum_{i=1}^{N_{s}} C_{s}\left(d, z_{i}, \theta_{i}\right)}{N_{s}}
$$

$$
E\left(C_{o}\right)=\frac{\int_{Z} C_{o}(z) I(z) d z}{\int_{Z} I(z) d z}=\frac{\sum_{i=1}^{N_{s}} C_{o}\left(z_{i}\right)}{N_{s}}
$$

The probability $P\left(g\left(d, z, y_{s}, \theta\right) \leq 0\right)$ is estimated by the ratio of the number of $g_{i}$ values less than or equal to zero to the total number of observations, $N_{s}$.

To compute the costs $C_{d}$ and $C_{z}$ sampling values are not needed: $C_{d}$ depends only on the deterministic values of the design variables, and the control $\operatorname{cost} C_{z}$ is determined from probability distribution parameters (for instance, the control cost associated with a single normal distributed variable may be expressed as a function of its mean and standard deviation estimates).

The overall objective function used is computed by summing the expected value estimates for $C_{s}$ and $C_{0}$ with equipment and control costs:

$$
C=E\left(C_{s}\right)+E\left(C_{o}\right)+C_{d}(d)+C_{z}(Z),
$$

where $C$ is itself an expected value for a total cost random variable that depends on the joint PDF $I J(z, \theta)$.

After the values for the objective function and restrictions (inequalities) have been calculated as stated above, an optimization procedure (NPSOL package, Gill et al., 1986) updates the values of the decision variables using information about partial derivatives of the objective function and restrictions (inequalities). Although the NPSOL algorithm is inherently deterministic, the generalized framework presented in this article is stochastic because the evaluation of the objective function accounts for the behavior of random variables.

The computational effort associated with this approach depends on the sampling technique chosen. We have applied the Hammersley sequence sampling (HSS) technique proposed by Diwekar and Kalagnanam $(1996,1997 \mathrm{a}, \mathrm{b})$, as it has been shown to be particularly efficient, reducing the number of observations required to come up with reliable objective function estimates. Subroutine SPARCLHS2 implements this and other sampling techniques, allowing the user to consider 


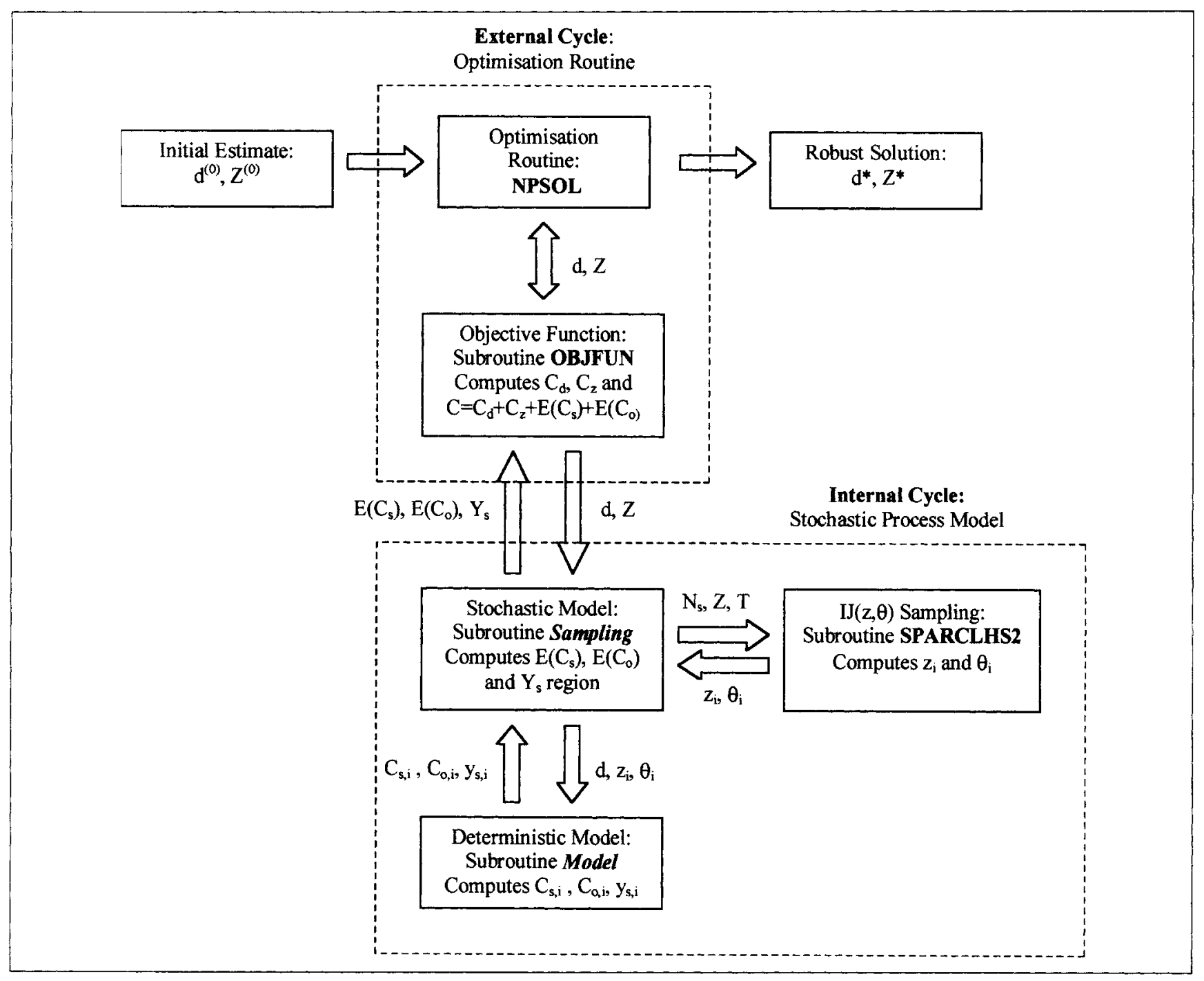

Figure 2. Robust optimization algorithm.

several kinds of input PDFs (independent or correlated, continuous or discrete).

The generic optimization approach presented so far allows almost any sort of process design problem subject to several kinds of uncertainty to be addressed.

Some issues related with the above optimization methodology deserve further discussion.

1) The external algorithm progression does not take into account the deterministic model as an explicit restriction. In fact, the deterministic model works as an implicit restriction because the objective function evaluation needs the output values, $y_{s, i}$, to be computed for all observations. If the deterministic model doesn't converge for a specific sample point, then the region $Z$ that includes that point is considered infeasible and the objective function (annual cost) is penalized with an infinite value, in order to guarantee that $P(h(d, z, y, \theta)=0)=1$.

2) Before starting the stochastic optimization procedure, the accuracy of the sampling technique used in the internal cycle must be evaluated. This is done by computing the number of observations, $N_{s}$, that are needed to predict the mean and standard deviation for the relevant outputs with enough accuracy (a maximum error of $1 \%$ is considered). Since $N_{s}$ depends on the process model used, sampling accuracy must be evaluated for each plant.

3) Computational effort depends directly on the value of $N_{s}$, since the total number for deterministic model evaluations that are needed is $N_{s} \times N_{i}+D$, where $N_{i}$ represents the number of iterations of the external optimization procedure and $D$ the number of evaluations needed to estimate partial derivatives. The HSS technique significantly reduces the magnitude of $N_{s}$ when compared with other techniques, such as MCS (Monte Carlo Sampling) or LHS (Latin Hypercube Sampling).

4) During the external algorithm progression we kept $N_{s}$ constant, although Chaudhuri and Diwekar $(1996,1997)$ have presented a "stochastic annealing algorithm" that uses increasing values of $N_{s}$ when the solution seems to approach optimality. When applied to process synthesis problems, this alternative has contributed to additional computational effort reduction and may also be explored within our optimization framework. 
5) The external algorithm procedure used in this article (NPSOL package) requires a large number of objective function evaluations (since partial derivatives are estimated by finite differences), fails to take full advantage of the information about the deterministic model obtained in each external iteration (for each objective function evaluation the deterministic model is applied $N_{s}$ times, and such values might possibly be explored in order to speed up and guide convergence), and does not offer global optimization guarantees.

The major goal of this article is to introduce a new robust optimization conceptual framework and problem formulation that has been applied and tested successfully over a small sample of relatively simple process models (which required CPU times on the order of minutes for finding an optimal solution). Therefore, we do not concentrate too much on algorithm or computational efficiency details or on comparing different optimization routines. The development of an external algorithm that assures a global optimum and tries to overcome some of the previous NPSOL limitations, together with applications to large scale problems, is currently the subject of further work, and will be reported in forthcoming publications.

\section{Case Studies}

To provide specific examples of application for the suggested robust design framework and methodology, we will consider two chemical processes. First, we will examine a simple model for a continuous stirred tank reactor (CSTR) subject to process-inherent uncertainty. Later, a batch distillation column will provide a more complex case study, subject to additional types of uncertainty.

\section{CSTR application}

In this section we will consider the CSTR already briefly introduced (Figure 1) with the nomenclature presented in Table 1.

Two first-order reactions in series $(A \rightarrow B \rightarrow C)$ with Arrhenius kinetics are assumed to occur. The stationary process model is obtained through an energy balance around the reactor and two material balances, referring to components $A$ and $B$ :

$$
\begin{aligned}
& \rho c_{p} F\left(T_{0}-T\right)+k_{A}^{0} \exp \left(-E_{A} / R T\right) C_{A}\left(-\Delta H_{1}\right) V \\
& +k_{B}^{0} \exp \left(-E_{B} / R T\right) C_{B}\left(-\Delta H_{2}\right) V-Q=0 \\
& F\left(C_{A 0}-C_{A}\right)-k_{A}^{0} \exp \left(-E_{A} / R T\right) C_{A} V=0 \\
& F\left(C_{B 0}-C_{B}\right)+k_{A}^{0} \exp \left(-E_{A} / R T\right) C_{A} V
\end{aligned}
$$

$$
-k_{B}^{0} \exp \left(-E_{B} / R T\right) C_{B} V=0
$$

The model variables can be classified according to our problem mathematical formulation (P):

$d=[V]$-design variable

$z=\left[F, Q, C_{A 0}, C_{B 0}, T_{0}\right]^{T}$-operating variables

$\theta=\left[E_{A}, E_{B}, k_{A}^{0}, k_{B}^{0}, \Delta H_{1}, \Delta H_{2}, \rho, c_{p}\right]^{T}$-process model parameters

$y_{s}=[P B]-$ quality variable
Table 1. CSTR Model Nomenclature

$\begin{aligned} V & =\text { reactor volume, } \mathrm{m}^{3} \\ F & =\text { volumetric flow rate, } \mathrm{m}^{3} / \mathrm{min} \\ Q & =\text { rate of heat removal, } \mathrm{J} / \mathrm{min} \\ C_{A 0}, C_{B 0} & =\text { inlet concentrations of } A \text { and } B, \mathrm{~mol} / \mathrm{m}^{3} \\ T_{0} & =\text { inlet temperature, } \mathrm{K} \\ C_{A}, C_{B} & =\text { concentrations in the reactor, } \mathrm{mol} / \mathrm{m}^{3} \\ T & =\text { reactor temperature, } \mathrm{K} \\ P B & =\text { rate of production of } B, \mathrm{~mol} / \mathrm{min} \\ E_{A}, E_{B} & =\text { activation energies, } \mathrm{J} / \mathrm{mol} \\ k_{A}^{0}, k_{B}^{0} & =\text { preexponential constants, } \mathrm{min}^{-1} \\ \Delta H_{1}, \Delta H_{2} & =\text { molar heats of the reactions, } \mathrm{J} / \mathrm{mol} \\ \rho & =\text { system density, } \mathrm{kg} / \mathrm{m}^{3} \\ c_{p} & =\text { system specific heat, } \mathrm{J} / \mathrm{kg} \mathrm{K} \\ R & =\text { ideal gas constant, } \mathrm{J} / \mathrm{mol} \mathrm{K}\end{aligned}$

The parameter values and the initial operating conditions are presented in Table 2. Process-inherent uncertainty is considered by describing all operating variables through independent normal probability density functions (these uncertainties are expressed using the operating window format in Table 2). The output quality-related variable is assumed to be the rate of production of $B, P B$, with a desired nominal value of $600 \mathrm{~mol} / \mathrm{min}\left(P B^{*}\right)$.

Our objective function is, as stated before, the overall annual plant cost, $C$, obtained from the following set of considerations (further details about cost functions can be found in Appendix A): equipment $\operatorname{cost}, C_{d}$, is the annually amortized reactor investment, expressed as a function of reactor volume $(V)$; operating costs are functions of both the flow rate, $F$, and the rate of heat removal, $Q$; and control costs, $C_{z}$, associated with each operating variable, $z$, are given by:

$$
C_{z}=\alpha+\beta \frac{\mu_{z}}{\sigma_{z}},
$$

where $\alpha$ and $\beta$ are constants, $\mu_{z}$ is the mean value of $z$, and $\sigma_{z}$ its standard deviation; quality costs are expressed by a symmetric Taguchi loss function:

$$
E\left(C_{s}\right)=k\left[\left(\mu_{P B}-P B^{*}\right)^{2}+\sigma_{P B}^{2}\right]
$$

where $k$ is a constant (quality loss coefficient).

The number of observations, $N_{s}$, needed to predict objective function values with enough accuracy is evaluated by increasing the number of observations employed until the mean and standard deviation for the output variable $P B$ can be

Table 2. CSTR Parameter and Initial Conditions

\begin{tabular}{ccccc}
\hline \multicolumn{2}{c}{ Parameters } & & \multicolumn{2}{c}{ Initial Operating Cond. } \\
\cline { 4 - 5 }$E_{A}$ & $3.64 \times 10^{4}$ & & $V$ & 0.391 \\
$E_{B}$ & $3.46 \times 10^{4}$ & & $F$ & $1.004(1 \pm 0.10)$ \\
$k_{A}^{0}$ & $8.4 \times 10^{5}$ & & $Q$ & $2.54 \times 10^{7}(1 \pm 0.10)$ \\
$k_{B}^{0}$ & $7.6 \times 10^{4}$ & & $C_{A 0}$ & $3,118(1 \pm 0.05)$ \\
$\Delta H_{1}$ & $-2.12 \times 10^{4}$ & & $C_{B 0}$ & $342.5(1 \pm 0.05)$ \\
$\Delta H_{2}$ & $-6.36 \times 10^{4}$ & & $T_{0}$ & $314(1 \pm 0.05)$ \\
$\rho$ & 1,180 & & $C_{A}$ & 2,476 \\
$c_{p}$ & $3.2 \times 10^{3}$ & & $C_{B}$ & 940.2 \\
$R$ & 8.314 & & $T$ & 311.6 \\
\hline
\end{tabular}



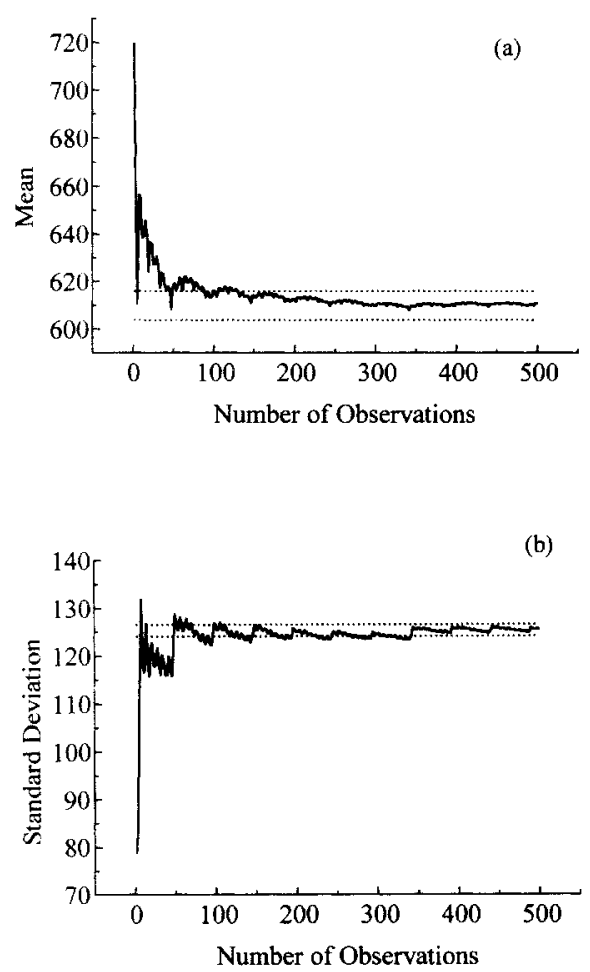

Figure 3. Mean (a) and standard deviation (b) PB estimates.

estimated with a maximum error of $1 \%$. Figure 3 shows the results obtained for the CSTR initial operating conditions, where a number of observations greater than 344 places the two represented quantities within the assumed error bands (dashed lines), therefore leading us to a conservative value of $N_{s}=400$.

To stress the relevance of considering different kinds of uncertainties and the associated robustness issues, Table 3 compares the results obtained under a fully deterministic approach, where all uncertainties are ignored, with the ones achieved by the proposed integrated robust optimization framework. Under a deterministic approach, the decision variables are the nominal values of the operating variables, while in our stochastic approach the $E_{z}$ tolerances are also considered as additional decision variables, besides the associated $\mu_{z}$ values. It can be seen that indeed both solutions are quite different and located in separate zones of the operating space. The robust solution defines the best operating region to implement a control system and the feed stream temperature, $T_{0}$, was identified as the critical control variable, for which a quite small tolerance must be achieved.

Table 3. Comparison Between the Deterministic and Robust Solution

\begin{tabular}{ccc}
\hline & Deterministic & Robust \\
\hline$V\left(\mathrm{~m}^{3}\right)$ & 0.3918 & 0.3463 \\
$F\left(\mathrm{~m}^{3} / \mathrm{min}\right)$ & 0.6621 & $0.5023(1 \pm 0.099)$ \\
$Q(\mathrm{~kJ} / \mathrm{min})$ & 1,619 & $146.7(1 \pm 0.080)$ \\
$C_{A 0}\left(\mathrm{~mol} / \mathrm{m}^{3}\right)$ & 3,449 & $3,140(1 \pm 0.050)$ \\
$C_{B 0}\left(\mathrm{~mol} / \mathrm{m}^{3}\right)$ & 34.25 & $510.7(1 \pm 0.050)$ \\
$T_{0}(\mathrm{~K})$ & 305.7 & $313.8(1 \pm 0.005)$ \\
\hline
\end{tabular}

Table 4. Outputs and Cost Associated with the Different Solutions

\begin{tabular}{crrr} 
& Determ. $^{*}$ & Determ.** $^{* *}$ & Robust \\
\hline & 608 & 602 & 600 \\
$\hat{\mu}_{P B}$ (mol/min) & 117 & 17.2 & 16.8 \\
$\hat{\sigma}_{P B}$ (mol/min) & 1,149 & 1,149 & 1,064 \\
$C_{d}$ (\$/year) & 1,145 & 2,105 & 2,105 \\
$C_{z}$ (\$/year) & 89,890 & 1,973 & 1,835 \\
$C_{s}$ (\$/year) & 9,688 & 9,688 & 9,712 \\
$C_{0}$ (\$/year) & 101,872 & 14,915 & 14,716 \\
$C$ (\$/year) &
\end{tabular}

* Tolerances of $10 \%$ ( $F$ and $Q$ ) and $5 \%\left(C_{A 0}, C_{B 0}\right.$, and $\left.T_{0}\right)$ as initial conditions, placed around deterministic optimum.

***optimal robust solution tolerances placed around the optimal deterministic pointwise solution.

In Table 4 we compare the outputs and the different costs obtained by the two approaches (deterministic and robust optimization), as well as the cost associated with placing the optimal robust solution tolerances around the deterministic optimal pointwise solution, thus building an operating region around it. The results obtained for the deterministic approach were obtained considering as tolerances the initial guesses used for stochastic programming (10\% for $F$ and $Q$, $5 \%$ for $C_{A 0}, C_{B 0}$, and $T_{0}$ ), and placing them around the optimal deterministic pointwise solution. The stochastic formulation corresponds to an expected output standard deviation that is approximately $14 \%$ of that obtained with the deterministic approach, the same percentage that occurs in the case of the overall average annual plant cost.

Figure 4 shows the optimal input PDFs that define the operating region $Z^{*}$. The PDFs for the output variable, $P B$, before and after robust optimization (Figure 5) illustrate the
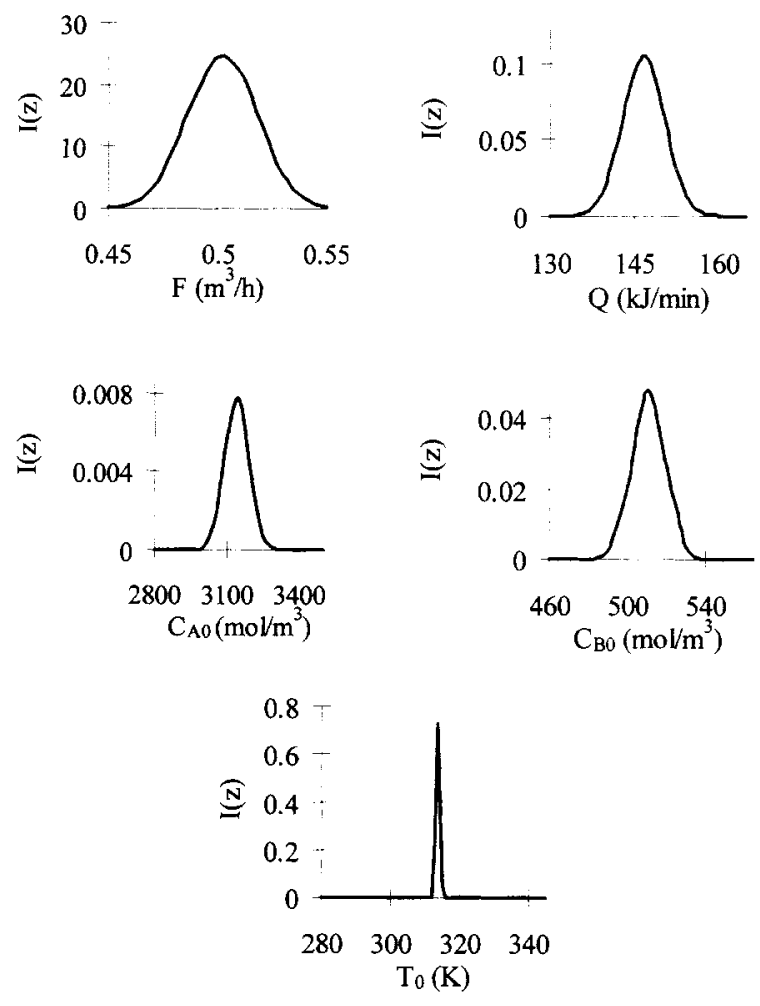

Figure 4. Optimal robust CSTR operating region. 


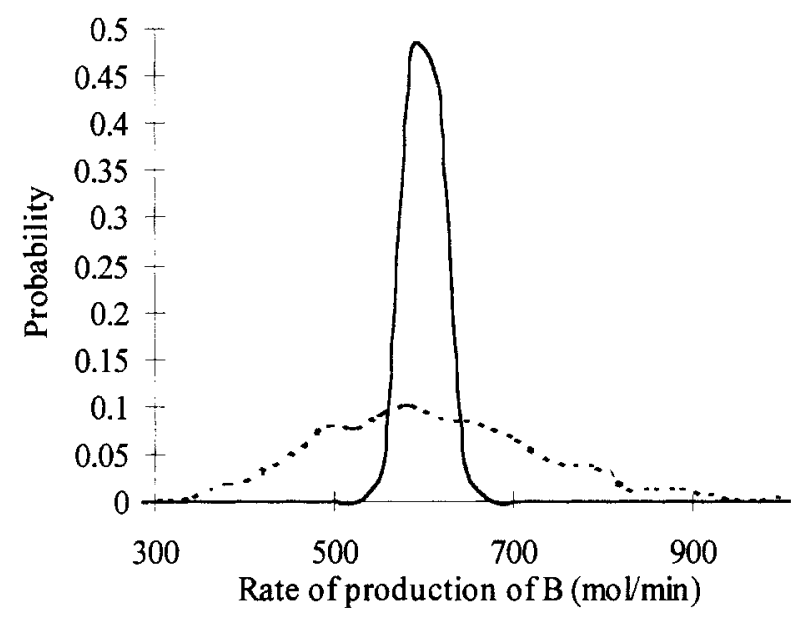

Figure 5. PB PDF before (broken line) and after (solid line) robust optimization.

quality improvement obtained when the stochastic methodology is used (initial values are the ones reported in Table 2).

\section{Batch distillation column application}

The batch distillation column (Figure 6) consists of a reboiler at the bottom, a rectifier column and a condenser at the top, with the nomenclature and initial operating conditions provided in Table 5 (we will only consider binary mixtures, with all the compositions mentioned referring to molar fractions on the more volatile component).

During batch time, the feed mixture becomes poorer in the more volatile component, and therefore $x_{D}$ decreases. In order to maximize the batch average product composition, $x_{D}^{*}$, the distillation column operates with time dependent reflux ratio and grows linearly with batch time:

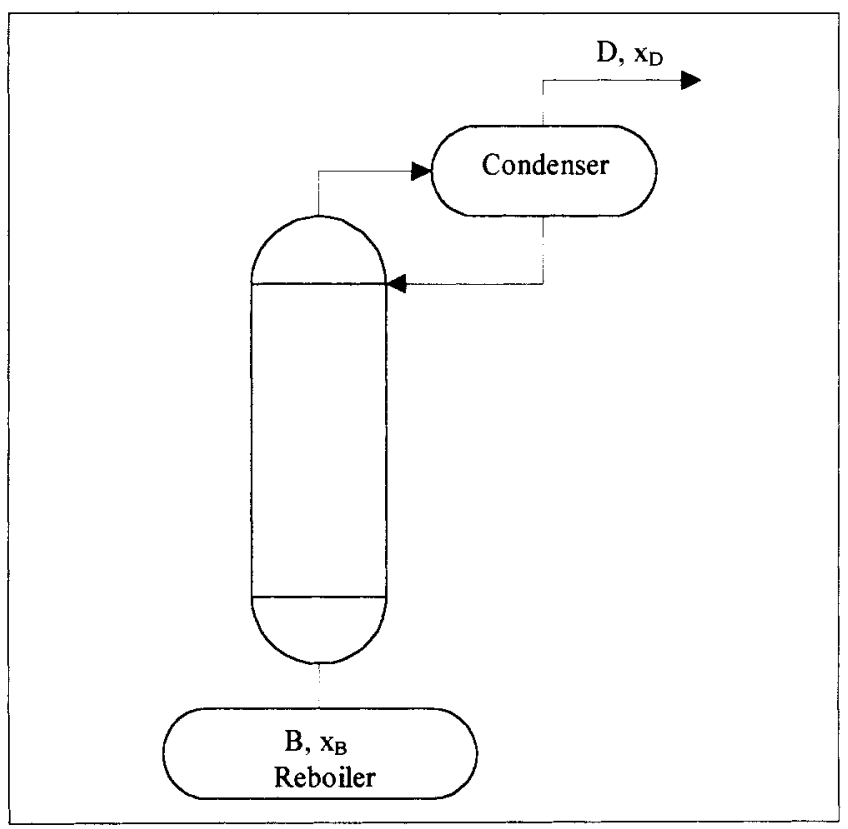

Figure 6. Batch distillation column.
Table 5. Batch Distillation Column Nomenclature and Initial Conditions

$\begin{array}{rlr}\alpha & =\text { relative volatility } & \\ N & =\text { number of theoretical stages } & \\ R & =\text { reflux ratio, } R(t)=R_{0}+R_{1} t & R(t)=2.3+t \\ V & =\text { vapor boilup rate, mol/h } & 100 \\ F & =\text { total feed, mol } & 100 \\ x_{F} & =\text { feed composition } & 0.5 \\ t & =\text { batch time, } \mathrm{h} & - \\ T & =\text { final total batch time, } \mathrm{h} & 0.7 \\ D & =\text { total distillate, mol } & 17.59 \\ x_{D} & =\text { product composition } & - \\ x_{D}^{*} & =\text { average product composition } & 0.9499 \\ B & =\text { bottom product, mol } & - \\ x_{B} & =\text { bottom composition } & \end{array}$

$$
R(t)=R_{0}+R_{1} t
$$

Model variables can be classified as follows, according to our problem mathematical formulation $(\mathrm{P})$ :

$d=[N]$ - design variable

$z=\left[R_{0}, R_{1}, V, F, x_{F}, T\right]^{T}$-operating variables

$\theta=[\alpha]$-process model parameter

$y_{s}=\left[x_{D}^{*}\right]$ - quality variable.

As output quality-related variable we will consider the average product composition, $x_{D}^{*}$, with a nominal desired value of 0.95 .

Our assumed objective function is the plant annual profit:

$$
L=L_{N}-C,
$$

where $L_{N}$ stands for the nominal revenue and $C$ represents overall annual cost of operating the plant.

The nominal revenue is computed taking into account the nominal sales value for the product relative to the cost of feed. As before, the overall annual cost, $C$, is the sum of equipment, operating, control and quality costs (details in Appendix A), where

- Equipment cost is a function of the number of theoretical stages, $N$, and the vapor boilup rate, $V$.

- Operating costs depend on the vapor boilup rate, $V$, and the average molar weight of the mixture, $M$.

- Control cost is computed by considering as control variables the vapor boilup ratio, $V$, and the reflux ratio (controlled by the initial value, $R_{0}$ ), with the associated $C_{z}$ values being given by Eq. 18 .

- Quality cost is expressed by an asymmetric quadratic Taguchi loss function, with a larger penalty associated with product that is poor on the more volatile component.

This example integrates different sorts of uncertainties, as defined by Pistikopoulos (1995). The purpose of the robust design problem formulation is to define a batch distillation column that is flexible enough to operate well with different feed mixtures and compositions. Therefore, a design scenario under high levels of uncertainty is assumed (Table 6):

- Thermodynamic relationship errors lead to uncertainties in the relative volatility-model-inherent uncertainty.

- Operating variables are also subject to uncertainties, and therefore initial reflux ratio, $R_{0}$; vapor boilup rate, $V$; total feed, $F$; and feed composition, $x_{F}$, are considered to be random variables. 
Table 6. Batch Distillation Column Uncertainties

\begin{tabular}{lc}
\hline Model-inherent & $\alpha=2(1 \pm 0.1)$ (uniform) \\
\hline Process-inherent & $R_{0}=2.3(1 \pm 0.1)$ (normal) \\
$V$ & $=100(1 \pm 0.1)$ mol/h (normal) \\
& $F=100(1 \pm 0.2)$ mol (uniform) \\
& $x_{F}=0.5(1 \pm 0.2)$ (uniform) \\
\hline External & $M=0.085(1 \pm 0.2) \mathrm{kg} / \mathrm{mol}$ (uniform) \\
\hline
\end{tabular}

- External uncertainties are also accounted for through the consideration of the mixture average molar weight as a parameter with a given probability density function.

As stated before, the accuracy of the sampling technique must be evaluated for each process model, with 500 observations needed in this case to reliably predict the $x_{D}^{*}$ mean and standard deviation.

The results obtained by the two distinct approaches are compared in Table 7 . In the deterministic formulation the decision variables are the number of theoretical stages, $N$; the reflux ratio (variables $R_{0}$ and $R_{1}$ ); the vapor boilup rate, $V$; and the batch time, $T$; whereas in the robust optimization tolerances for the control variables $V$ and $R_{0}$ are also treated as decision variables. The optimal robust solution is once again located in a quite different zone of the decision space, indicating $V$ as the control variable where a smaller tolerance should be achieved (Figure 7). When we attempted to place optimal robust tolerances around the deterministic pointwise solution, the resulting region was found to be infeasible (the batch distillation column model does not converge for at least one observation). This fact, by itself, reinforces the relevance of the stochastic approach and points out the limitations of fully deterministic solutions that do not account for important uncertainty sources that affect plant operation.

Table 8 compares the outputs and costs before and after stochastic optimization (the initial values are the ones reported in Table 5, with the uncertainty scenario of Table 6). The optimization procedure results in a reduction of $35 \%$ in the annual plant cost and a $13 \%$ increase in annual profit. The output PDF for average product composition moves in the direction of richer product and its standard deviation is reduced from 0.0318 to 0.0290 (Figure 8), resulting in a $48 \%$ decrease of quality cost. This output variability reduction is achieved while control tolerances increase significantly $\left(R_{0}\right)$ or slightly $(V)$, indicating that the space region identified leads to better performance and was able to do so together with easier to achieve process control goals (Figure 8).

\section{Conclusions}

We have developed a robust optimization framework that is able to integrate different sorts of uncertainties, control,

Table 7. Comparison Between Deterministic and Robust Solutions

\begin{tabular}{ccc}
\hline & Deterministic & Robust \\
\hline$N$ & 15.74 & 11.63 \\
$R_{\mathrm{i}}$ & 2.063 & $2.058(1 \pm 0.181)$ \\
$R_{1}$ & 0.6622 & 1.690 \\
$V(\mathrm{~mol} / \mathrm{h})$ & 122.2 & $107.2(1 \pm 0.0988)$ \\
$T(\mathrm{~h})$ & 0.5000 & 0.6912 \\
\hline
\end{tabular}
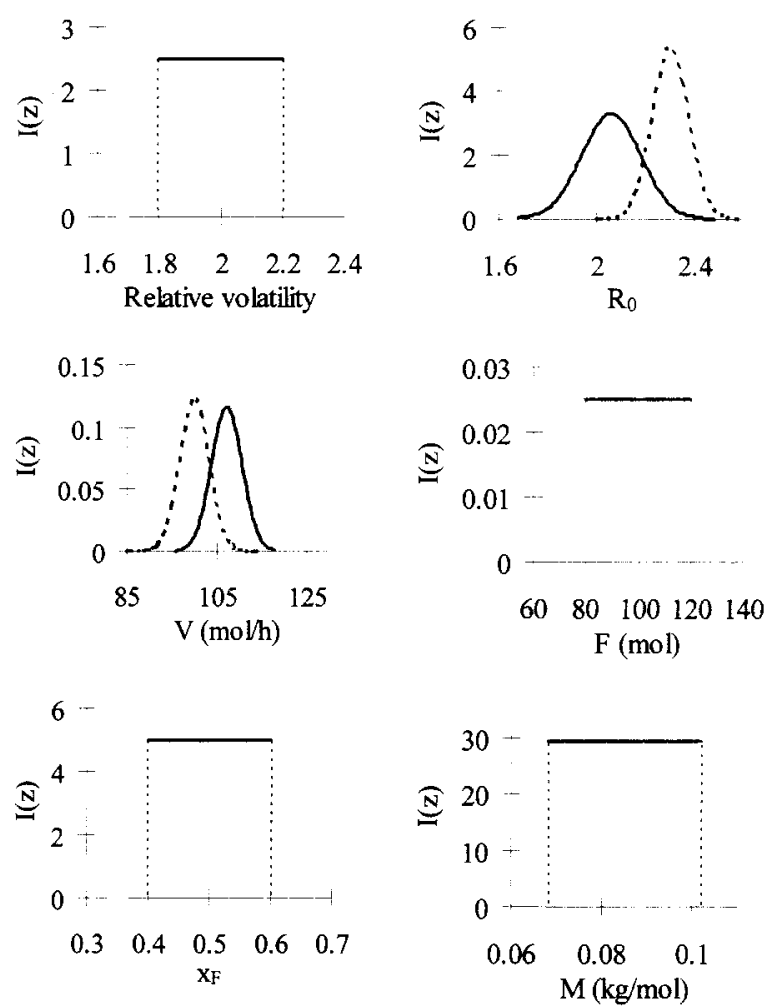

Figure 7. Parameter uncertainty and operating regions for the distillation column: initial conditions (broken line) and after robust optimization (solid line).

quality, and operation issues at an early plant design stage, leading to the simultaneous definition of optimal system, parameter, and tolerance designs as defined by Taguchi (1986). Optimal operating zones are identified together with associated control needs, providing a solution that takes into account investment, quality, operating, and control costs.

The mathematical formulation developed leads to a stochastic optimization problem solved by coupling a stochastic process model with an external optimization procedure. An efficient sampling technique is used to estimate objective function expected values.

The suggested approach, and the solution methodology associated with it, can be applied to almost any sort of process design problem. Two specific examples were addressed in this

Table 8. Outputs and Costs Associated with Initial Conditions and Robust Solution

\begin{tabular}{ccc}
\hline & Initial Conditions & Robust Solution \\
\hline$\hat{\mu}_{D}($ mol $)$ & 17.62 & 17.68 \\
$\hat{\sigma}_{D}($ mol $)$ & 0.6512 & 0.7524 \\
$\hat{\mu}\left(x_{D}^{*}\right)$ & 0.9416 & 0.9667 \\
$\hat{\sigma}\left(x_{D}^{*}\right)$ & 0.0318 & 0.0290 \\
\hline$C_{d}(\$ /$ year $)$ & 196 & 244 \\
$C_{z}$ (\$/year) & 394 & 370 \\
$C_{s}$ (\$/year) & 3,605 & 1,879 \\
$C_{o}$ (\$/year) & 611 & 654 \\
$C$ (\$/year) & 4,806 & 3,147 \\
$L_{N}$ (\$/year) & 19,298 & 19,571 \\
$L$ (\$/year) & 14,492 & 16,424 \\
\hline
\end{tabular}




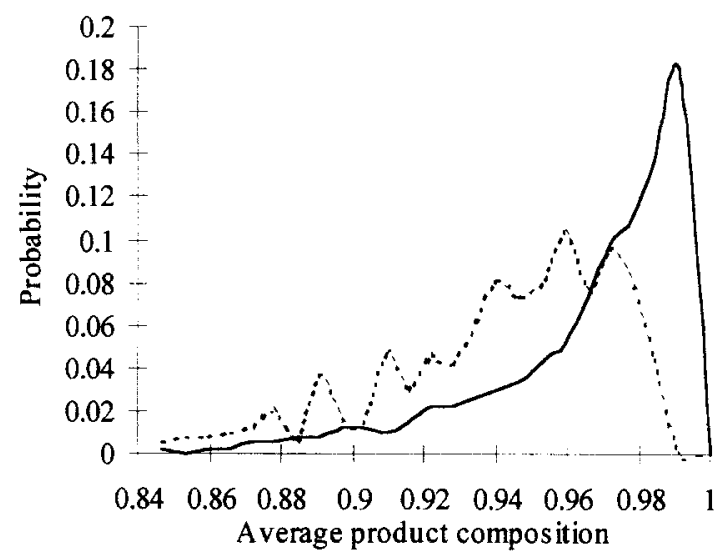

Figure 8. $x_{D}^{*}$ PDF before (broken line) and after (solid line) robust optimization.

article (a CSTR and a batch distillation column), providing enough evidence for the usefulness and relevance of both our problem formulation and solution methodology.

\section{Acknowledgments}

The authors wish to acknowledge all the support (including unpublished materials and code) provided by Urmila Diwekar (Carnegie Mellon University).

\section{Literature Cited}

Bahri, P. A., J. Bandoni, and J. Romagnoli, "Effect of Disturbances in Optimizing Control: Steady-State Open-Loop Backoff Problem," AIChE J., 42, 983 (1996).

Bahri, P. A., J. Bandoni, and J. Romagnoli, "Integrated Flexibility and Controllability Analysis in Design of Chemical Processes," AIChE J., 43, 997 (1997).

Chaudhuri, P. D., and U. M. Diwekar, "Process Synthesis under Uncertainty: A Penalty Function Approach," AIChE J., 42, 742 (1996).

Chaudhuri, P. D., and U. M. Diwekar, "Synthesis under Uncertainty with Simulators," Comp. Chem. Eng., 21, 733 (1997).

Czitrom, V., and P. Spagon, Statistical Case Studies for Industrial Process Improvement, SIAM, Philadelphia (1997).

Diwekar, U. M., and J. R. Kalagnanam, "Robust Design Using an Efficient Sampling Technique," Comp. Chem. Eng., 20, S389 (1996).

Diwekar, U. M., and J. R. Kalagnanam, "An Efficient Sampling Technique for Optimization Under Uncertainty," AIChE J., 43, 440 (1997a).

Diwekar, U. M., and J. R. Kalagnanam, "An Efficient Sampling Technique for Off-line Quality Control," Technometrics, 39, 308 (1997b)

Diwekar, U. M., and E. S. Rubin, "Stochastic Modeling of Chemical Processes," Comp. Chem. Eng., 15, 105 (1991).

Diwekar, U. M., and E. S. Rubin, "Parameter Design Methodology for Chemical Processes Using a Simulator," Ind. Eng. Chem. Res., 33, 292 (1994).

Douglas, J. M., Conceptual Design of Chemical Processes, McGrawHill, New York (1988).

Gill, P. E., W. Murray, M. A. Saunders, and M. H. Wright, "User's Guide for NPSOL: A Fortran Package for Nonlinear Programming," Technical Report SOL 86-2, Systems Optimization Laboratory, Stanford University, Stanford, California (1986).

Grossmann, I. E., and C. A. Floudas, "Active Constrain Strategy for Flexibility Analysis in Chemical Processes," Comp. Chem. Eng., 11, 675 (1987).

Luyben, M. L., and C. A. Floudas, "Analysing the Interaction of Design and Control-1. A Multiobjective Framework and Application to Binary Distillation Synthesis," Comp. Chem. Eng., 18, 933 (1994).
Mohideen, M. J., J. Perkins, and E. Pistikopoulos, "Optimal Design of Dynamic Systems under Uncertainty," AIChE J., 42, 2251 (1996).

Peters, M. S., and K. D. Timmerhaus, Plant Design and Economics for Chemical Engineers, 4th ed., McGraw-Hill, New York (1991).

Phadke, M. S., Quality Engineering using Robust Design, Prentice Hall, Englewood Cliffs, NJ (1989).

Pistikopoulos, E. N., "Uncertainty in Process Design and Operations, Comp. Chem. Eng., 19, S553 (1995).

Pistikopoulos, E. N., and M. G. Ierapetritou, "Novel Approach for Optimal Process Design under Uncertainty," Comp. Chem. Eng., 19. 1089 (1995).

Saraiva, P., and G. Stephanopoulos, "Continuous Process Improvement Through Inductive and Analogical Learning," AIChE J., 38, 161 (1992)

Saraiva, P., "Data-Driven Learning Frameworks for Continuous Process Analysis and Improvement," PhD Thesis, Massachusetts Institute of Technology, Cambridge, MA (1993).

Saraiva, P., "Inductive and Analogical Learning: Data-Driven Improvement of Process Operations," in Intelligent Systems in Process Engineering: Paradigms from Design and Operations, G. Stephanopoulos and C. Han, eds., p. 377-435, Academic Press, New York (1996).

Schmidt, S., and R. Launsby, Understanding Industrial Designed Experiments, Air Academy Press, Colorado Springs, CO (1992).

Straub, D. A., and I. E. Grossmann, "Integrated Stochastic Metric of Flexibility for Systems with Discrete States and Continuous Parameter Uncertainties," Comp. Chem. Eng., 14, 967 (1990).

Straub, D. A., "Evaluation and Optimization of Process Systems with Discrete and Continuous Uncertainties," Ph.D. Thesis, CarnegieMellon Univ., Pittsburgh, PA (1991).

Swaney, R. E., and I. E. Grossmann, "An Index for Operational Flexibility in Chemical Process Design. I: Formulation and Theory,' AIChE J., 31, 621 (1985).

Taguchi, G., Introduction to Quality Engineering, Amer. Supplier Inst., Dearborn, MI (1986).

Taylor, W., Optimization and Variation Reduction in Quality, McGraw-Hill, New York (1991).

\section{Appendix: Cost Functions}

\section{CSTR application}

The assumed objective function is the overall annual plant cost, $C$, which includes the four cost categories: equipment $\operatorname{cost}, C_{d}$, operating cost, $C_{o}$, control cost, $C_{z}$, and quality cost, $C_{s}$.

Douglas (1988) presents an installation cost correlation for cylindrical reactors as a function of the diameter, $D(\mathrm{ft})$, and the height, $H(\mathrm{ft})$ :

$$
C_{d}=\left(\frac{\mathrm{M} \& \mathrm{~S}}{280}\right) 101.9 D^{1066} H^{0.802}\left(2.18+F_{c}\right)
$$

where $F_{c}=2$ includes material and operating pressure indexes. Considering $D=H, \mathrm{M} \& \mathrm{~S}=900$ and a three-year constant amortization, we have:

$$
C_{d}, \$ / \mathrm{yr}=4.18\left(\frac{900}{280}\right)\left(\frac{937.7}{3}\right)\left(\frac{V}{\pi}\right)^{0.6227},
$$

with the volume expressed in $\mathrm{m}^{3}$.

The operating costs include heating/refrigeration costs:

$$
C_{\text {util }}, \$ / \mathrm{yr}=\frac{900}{786}\left(7,896-6,327 q+4.764 \times 10^{4} q^{2}\right.
$$

$$
\left.-1.022 \times 10^{4} q^{4}\right),
$$


where $q=Q / Q^{N}$ and $Q^{N}=2.54 \times 10^{7} \mathrm{~J} / \mathrm{min}$; and pumping costs:

$$
C_{\text {pump }}, \$ / \mathrm{yr}=\left(\frac{900}{834}\right)\left(\frac{38.60}{3}\right)(264.2 F)^{0.8050}
$$

with $F$ expressed in $\mathrm{m}^{3} / \mathrm{min}$. Equation $\mathrm{A} 3$ results from a polynomial regression based on values presented by Douglas (1988); Eq. A4 is based on an installation cost graphic correlation for centrifugal pumps (Peters, 1991).

Control costs are estimated by Eq. 18. The residual cost, $\alpha$, is considered as $6 \%$ of $C_{d}+C_{\text {pump }}$ for the initial operating conditions (Table 2), which give $\alpha=\$ 143.2$. The $\beta$ parameter is computed considering the control cost for a single operating variable as $7.5 \%$ of $C_{d}+C_{\text {pump }}$ (for the initial operating conditions), when the variable tolerance is $E_{z}=0.15$, leading to $\beta=1.736$. The total control cost is the sum of the contributions corresponding to the five operating variables:

$$
C_{z}, \$ / \mathrm{yr}=5 \alpha+\beta\left(\frac{\mu_{F}}{\sigma_{F}}+\frac{\mu_{Q}}{\sigma_{Q}}+\frac{\mu_{C_{A 0}}}{\sigma_{C_{A 0}}}+\frac{\mu_{C_{B 0}}}{\sigma_{C_{B 0}}}+\frac{\mu_{T_{0}}}{\sigma_{T_{0}}}\right)
$$

Finally, quality costs are expressed by the symmetric Taguchi loss function Eq. 19, where the constant $k$ is computed assuming that a $10 \%$ deviation on the quality-related variable $P B$ leads to an increase of $50 \%$ in the cost $C_{d}+C_{o}$ (for the initial operating conditions). This assumption results in a $k$ value of 6.536 .

\section{Batch distillation column application}

Our objective function is the plant annual profit given by Eq. 21.

Equipment, operating costs, and control costs are given by the following expressions:

$$
C_{d}, \$ / \mathrm{yr}=\frac{c_{1} V M P}{G_{a}}+\frac{c_{2} V}{G_{b}},
$$

where $P$ is the number of theoretical plates $(P=N-1), c_{1}$ $=\$ 15 / \mathrm{m}^{2} /$ (theoretical plate) $/ \mathrm{yr}, c_{2}=1.65 \$ / \mathrm{m}^{2} / \mathrm{yr}$ and $G_{a}=$
$15 \mathrm{~kg} / \mathrm{h} / \mathrm{m}^{2}$ (Diwekar and Kalagnanam, 1997a,b);

$$
C_{0}, \$ / \mathrm{yr}=\frac{24(365) c_{3} V M T}{T+t_{s}},
$$

where $c_{3}=0.00935 \$ / \mathrm{kg}$ and $t_{s}=0.1 \mathrm{~h}$ is the stop time between batches (Diwekar and Kalagnanam, 1997a,b);

$$
C_{z}, \$ / \mathrm{yr}=2 \alpha+\beta\left(\frac{\mu_{R_{0}}}{\sigma_{R_{0}}}+\frac{\mu_{V}}{\sigma_{V}}\right) \text {, }
$$

where $\alpha$ and $\beta$ have the same values as for the CSTR example: $\alpha=\$ 143.2$ and $\beta=1.736$.

The nominal profit is based on the nominal sales value for the product relative to the cost of feed, $P_{r}^{N}=0.1 \$ / \mathrm{mol}(\mathrm{Di}$ wekar and Kalagnanam, 1997a,b):

$$
L_{N}, \$ / \mathrm{yr}=\frac{24(365) D P_{r}^{N}}{T+t_{s}},
$$

When the average product composition differs from the desired value $(0.95)$, the product sales price, $P_{r}$, is penalized through an asymmetric quadratic Taguchi loss function. For a given observation $i$, corresponding to an average product composition $x_{D, i}^{*}$, the penalty function is:

$$
P_{r, i}=\left\{\begin{array}{l}
P_{r}^{N}-k_{1}\left(x_{D, i}^{*}-0.95\right)^{2} \Leftarrow x_{D, i}^{*} \leq 0.95 \\
P_{r}^{N}-k_{2}\left(x_{D, i}^{*}-0.95\right)^{2} \Leftarrow x_{D, i}^{*}>0.95,
\end{array}\right.
$$

where $k_{1}=20$ and $k_{2}=4$. The associated quality cost is given by:

$$
C_{s, i}, \$ / \mathrm{yr}=\frac{24(365) D\left(P_{r}^{N}-P_{r, i}\right)}{T+t_{s}}
$$

Manuscript received Feb. 23, 1998, and revision received June 20, 1998. 